\title{
Development of a novel microbubble-liposome complex conjugated with peptide ligands targeting IL4R on brain tumor cells
}

\author{
SEE-HYOUNG PARK ${ }^{1,3,7}$, YOUNG II YOON ${ }^{1,3}$, HYOUNGWON MOON $^{3}$, GA-HYUN LEE $^{4}$, \\ BYUNG-HEON LEE ${ }^{5}$, TAE-JONG YOON ${ }^{6}$ and HAK JONG LEE ${ }^{1-3}$
}

\begin{abstract}
${ }^{1}$ Program in Nano Science and Technology, Department of Transdisciplinary Studies, Seoul National University, Graduate School of Convergence Science and Technology, Suwon; ${ }^{2}$ Department of Radiology, Seoul National University, College of Medicine, Seoul; ${ }^{3}$ Department of Radiology, Seoul National University, Bundang Hospital, Seungnam, Republic of Korea; ${ }^{4}$ Department of Cellular and Molecular Biology, California State University, Chico, CA, USA; ${ }^{5}$ Department of Biochemistry and Cell Biology, Kyungpook National University, Daegu;

${ }^{6}$ Department of Pharmacy, Ajou University, Suwon, Republic of Korea
\end{abstract}

Received December 1, 2015; Accepted January 5, 2016

DOI: $10.3892 /$ or.2016.4836

\begin{abstract}
Gas (SF6)-filled microbubbles (MBs) were prepared by emulsion and solvent-evaporation method. The prepared MBs were further conjugated with doxorubicin (Dox)-loaded nano-sized liposome and peptide ligands to interleukin-4 receptor (IL4R) for targeting brain tumor cells. The final MB-liposome (Dox)-IL4R targeting peptide ligand [MB-Lipo (Dox)-IL4RTP] had a spherical structure with the mean size of 1,500 nm. The MB-Lipo (Dox)-IL4RTP exhibited cellular uptake in U87MG brain tumor cells (a brain tumor cell line expressing strongly IL4R) with frequency ultrasound energy suggesting that MB-Lipo (Dox)-IL4RTP provided effective targeting ability for brain tumor cells. In addition, WST-1 assay results showed that MB-Lipo (Dox)-IL4RTP inhibited the proliferation of U87MG cells IL4R-dependently. This was confirmed by western blotting of $\gamma \mathrm{H} 2 \mathrm{AX}$, phospho (Ser15)-p53,p53 and p21 which are signal transduction proteins involved in DNA damage response and cell cycle arrest. Taken together, these results indicate that MB-Lipo (Dox)-IL4RTP represents a promising ultrasonic contrast agent for tumortargeting ultrasonic imaging.
\end{abstract}

\section{Introduction}

Brain tumor develops from glial or precursor cells. There are several types of brain tumors including astrocytoma,

Correspondence to: Dr Hak Jong Lee, Department of Radiology, Seoul National University, College of Medicine, Seoul, Republic of Korea

E-mail: hakjlee@snu.ac.kr

Present address: ${ }^{7}$ Department of Bio and Chemical Engineering, Hongik University, Sejong 339-701, Republic of Korea

Key words: microbubble, liposome, ligand, IL4R, brain, tumor oligodendroglioma, glioblastoma (GBM), ependymoma, malignant and mixed glioma, and not otherwise specified (NOS). GBM is one of the neuroepithelial tissue tumors and sub-classified into gliosarcoma and giant-cell GBM. In adults, GBM occurs most commonly in the primary malignant brain tumor. In general, GBM represent $17 \%$ of all types of brain tumors including both primary and metastatic state. Adults from the age of 45 to 70 tend to be diagnosed with these tumors. The average age of death from brain and other nervous system cancer is at the of age of 64 years. GBM has an incidence of $3.19 / 100,000$ in the US $(1,2)$ and $\sim 0.59-3.69$ cases $/ 100,000$ are annually diagnosed worldwide (3-7). It is one of the most devastating glial tumors that typically results in survival of only one-third of patients in the first year, and less than $5 \%$ beyond 5 years after diagnosis (8). The median survival with GBM is $\sim 12-15$ months despite the aggressive surgical and conventional treatment $(9,10)$.

Interleukin (IL) 4 acts through a multi-unit transmembrane receptor, IL4R. The overexpression of IL4R has been observed in brain, head and neck, lung, pancreatic, prostate cancers, and AIDS-associated Kaposi's sarcoma (11-14), whereas normal cells including immune cells express a low number of this receptor $(15,16)$. IL4R complex presented (in cells) are of two different types. Type I IL4R consists of IL4R $\alpha$ and IL2R $\gamma$ chains; however, type II IL4R has IL4R $\alpha$ and IL13R $\alpha 1$ chains. The signal transduction through type I or II IL4R is mediated by two receptor chains (17). It was also observed that the blockade of IL4R abrogates highly both hematogenous and lymphatic metastases in vivo and therefore, IL4R signaling pathway could be a target for inhibiting rhabdomyosarcoma tumor progression and metastasis (18).

Although the detail biological function of IL4R expression on brain tumors is not clearly understood, based on previous studies, it seems that IL4R expression may play an important role in brain tumor development and be one of the potential candidate cell surface target proteins for a targeted diagnostic and therapy. As one of the novel approach for this purpose, 
peptide drugs have been applied to treat tumors with the high IL4R expression since peptides can be relatively easily synthesized by either biological recombinant method or chemical solid phase synthesis. In addition, costs for production are less than for antibody-based therapeutics (19). IL4R $\alpha$ has been previously reported to have high expression on the surface of a variety of human solid tumors such as renal cell carcinoma, malignant melanoma and GBM (20).

In the present study IL4R targeting peptide ligandconjugated liposomal microbubbles [(MBs)-Lipo doxorubicin (Dox)-IL4RTP] were prepared as ultrasound contrast agents, and to analyze the anticancer effect of the novel ultrasound contrast agents containing MBs in vitro in the cell line model of brain tumor, which is a clinically significant tumor to provide experimental proof of concept for specific ultrasound imaging of brain tumor at an early stage.

\section{Materials and methods}

Chemical reagents and antibodies. Dimethyl sulfoxide (DMSO), glycerol, glycine, sodium chloride, thiazolyl blue tetrazolium bromide, Trizma-base and Tween-20 were purchased from Sigma (St. Louis, MO, USA). IL4R targeting peptide ligand (sequence: CRKRLDRN) was from Anygen Inc. (Daejeon, Korea). Mouse anti-p53, mouse anti-PARP1, rabbit anti-IL4R and mouse anti- $\beta$-actin antibodies were obtained from Santa Cruz Biotechnology (Santa Cruz, CA, USA). Rabbit anti-Bax (1:1,000 dilution), rabbit anti-phospho-p53 (Ser15), rabbit anti-p21 and rabbit anti- $\gamma \mathrm{H} 2 \mathrm{AX}$ antibodies were purchased from Cell Signaling Technology (Danvers, MA, USA). Goat anti-mouse and goat anti-rabbit horseradish peroxidase-conjugated IgG were obtained from Jackson ImmunoResearch (West Grove, PA, USA). ECL Western Blotting detection reagents were obtained from GenDEPOT (Barker, TX, USA).

Cell culture. U87MG, LN229, HS683, U138, MDA-MB-231 and $\mathrm{H} 460$ cells [all from American Type Culture Collection (ATCC); Manassas, VA, USA) were maintained in Dulbecco's modified Eagle's medium (DMEM) media supplemented with $10 \%$ fetal bovine serum and $1 \%$ streptomycin/penicillin at $37^{\circ} \mathrm{C}$ in a humidified incubator containing $5 \% \mathrm{CO}_{2}$ in air.

Preparation of MB-Lipo complex. The protocol for preparing of MB-Lipo complex was based on our previous study (21). Thiol-active MBs $(0.67 \mathrm{ml})(13 \mathrm{mg} / \mathrm{ml}, 1.2 \times 1,011 \mathrm{MB} / \mathrm{ml})$, derived from DSPE-PEG-PDP, were mixed with $2 \mathrm{ml}$ thiolated Lipo $(10 \mathrm{mg} / \mathrm{ml})$ by shaking for $2 \mathrm{~h}$ at room temperature. The reaction was monitored to pyridine-2-thione releasing by UV-Vis spectra. The prepared Lipo still had an amine functional group on the surface, despite treatment with Traut's reagent, as shown by quantitative amine analysis. The $30 \mathrm{mg}$ MB-Lipo dispersion aqueous solution (2 ml), containing an amine functional group, was reacted with sulfosuccinimidyl-4-(N-maleimidomethyl) cyclohexane1-carboxylate (Sulfo-SMCC; $5 \mathrm{mg}$; Sigma-Aldrich) for $3 \mathrm{~h}$ at $25^{\circ} \mathrm{C}$ after adjusting the $\mathrm{pH} \sim 8.2$ with $1 \mathrm{M} \mathrm{NaHCO}_{3}$, to activate maleimide functional group. This particle solution was then linked to appropriate antibodies using a general conjugation procedure.
Dox loading into MB-Lipo. Dox (Sigma-Aldrich) was incorporated into Lipo particles by the thin-film hydration and remote-loading method with an ammonium sulfate gradient. Lipid film (21.1 mg) was hydrated with $2 \mathrm{ml}$ ammonium sulfate solution $(250 \mathrm{mM})$, and the liposomal suspension was sequentially extruded 5 times through polycarbonate filters with pore sizes of $200 \mathrm{~nm}$. The ammonium sulfate was removed by centrifugation ( $5 \mathrm{~min}, 13,000 \mathrm{rpm}$ ). At this point, ammonium sulfate ions were located only inside of Lipo particles. The liposomal dispersion and $440 \mu \mathrm{M}$ Dox $(1: 1, \mathrm{v} / \mathrm{v})$ were mixed and incubated for $2 \mathrm{~h}$ at $60^{\circ} \mathrm{C}$. The mixture was washed to remove unloaded Dox, and the loading capacity of Dox in Lipo was determined by measuring the concentration of Dox in the supernatant by UV-Vis spectroscopy. LipoDox particles were then complexes with MBs as described above.

Preparation and characterization of MB-Lipo (Dox)-IL4RTP. MB-Lipo (Dox) complex containing NH2 group was reacted with Sulfo-SMCC in phosphate-buffered saline (PBS; $\mathrm{pH}$ 8.2) for $2 \mathrm{~h}$ to introduce maleimide group outside the MB-Lipo (Dox) complex. Then, MB-Lipo (Dox)-maleimide was further conjugated with IL4R targeting peptide-thiol group in PBS (pH 7.5) for $2 \mathrm{~h}$ to produce MB-Lipo (Dox)-IL4RTP. For determining the conjugation reaction of the IL4R targeting peptide into MB-Lipo complex, analytical high-performance liquid chromatography (HPLC) was adopted. Using water symmetry C18 column (4.6x220 nm), the eluting solvent was used for the $40 \%$ acetonitrile. At flow rate of the solvent $0.8 \mathrm{ml} /$ min a single absorption peak at $250 \mathrm{~nm}$ was identified.

Confocal microscopy analysis. The same quantity of IL4R targeting peptide ligand conjugated MB (FITC)-Lipo (Cy5) were added to U87MG cells with or without ultrasound flash (Sonidel SP-100; Sonidel, Boston, MA, USA), and then observed using confocal laser scanning microscopy. U87MG cells were grown on glass coverslips. After incubation with $\mathrm{MB}$ (FITC)-Lipo (Cy5) for $3 \mathrm{~h}$, cells were fixed with $4 \%$ paraformaldehyde for $10 \mathrm{~min}$ and permeabilized with Triton X-100 $(0.5 \%)$. Slide culture chambers were washed with PBS. Cells were stained with 4',6-diamidino-2-phenylindole (DAPI; Sigma) to show the nuclei. Specific staining was visualized and images were captured with confocal laser scanning microscope (Zeiss LSM510 microscope).

WST-1 cell viability assay. A $200 \mu 1$ aliquot of cells $\left(1 \times 10^{3}\right.$ cells in media) was added to each well of a 96-well plate and incubated for $18 \mathrm{~h}$ at $37^{\circ} \mathrm{C}$ in a humidified incubator containing $5 \% \mathrm{CO}_{2}$ in air. After incubation, MB-Lipo (Dox)-IL4RTP was added with or without ultrasound flash (Sonidel SP-100) into each well for $48 \mathrm{~h}$. Control cultures were treated with PBS. After incubation, a $20 \mu \mathrm{l}$ WST-1 solution was added to each well and the incubation continued for $4 \mathrm{~h}$. The visible absorbance at $560 \mathrm{~nm}$ of each well was quantified using a microplate reader.

Western blot analysis. Cells were washed with PBS and lysed in lysis buffer (50 mM Tris- $\mathrm{HCl}, 150 \mathrm{mM} \mathrm{NaCl}, 2 \mathrm{mM}$ EDTA, $1 \%$ Triton $\mathrm{X}-100,0.1 \%$ SDS, $\mathrm{pH}$ 8.0) with protease and phosphatase inhibitors. Cell lysates were centrifuged $(10,000 \mathrm{x} \mathrm{g}$, $\left.4^{\circ} \mathrm{C}, 10 \mathrm{~min}\right)$ and the supernatants were separated on 6 or $10 \%$ SDS-PAGE gels and blotted onto nitrocellulose membranes 

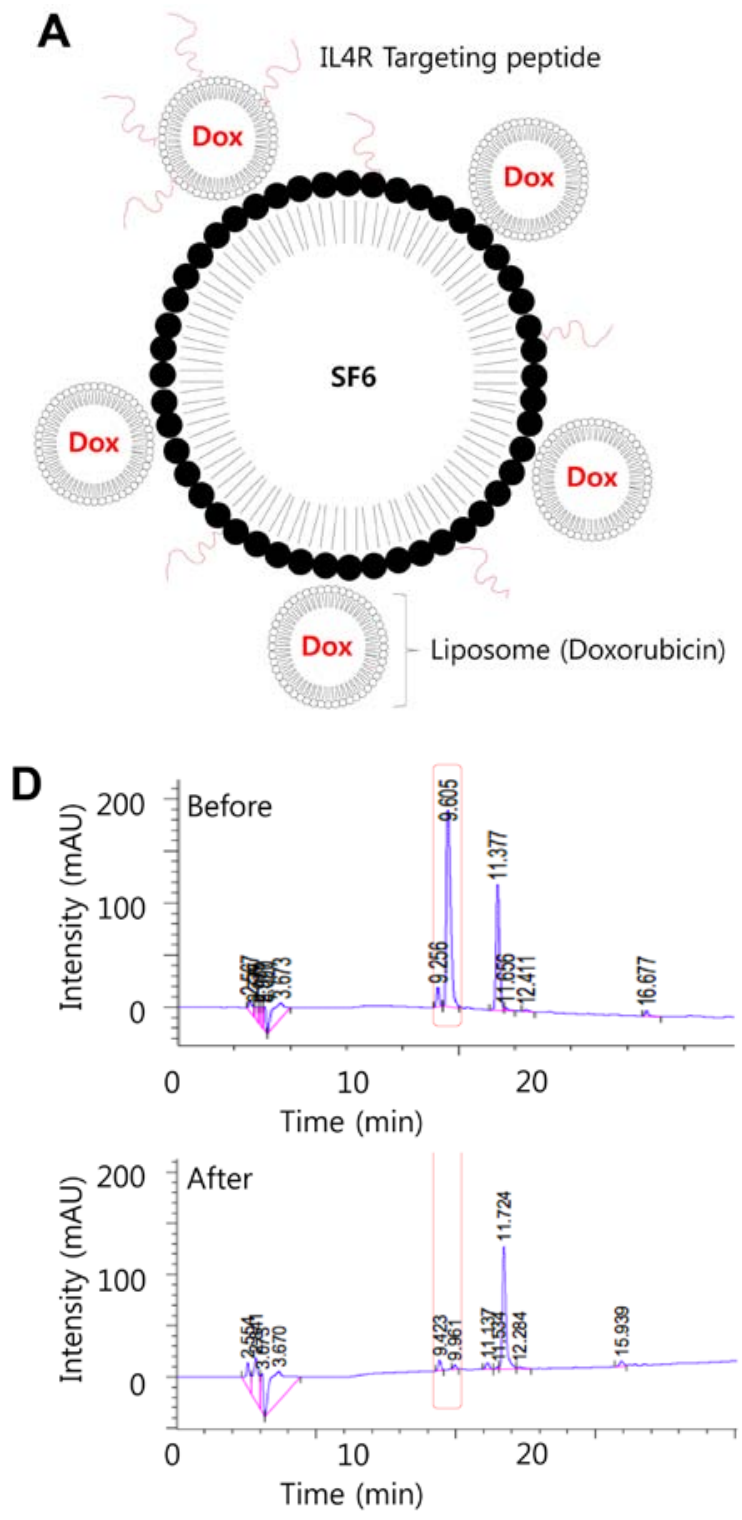

(Bio-Rad Laboratories, Hercules, CA, USA). The membranes were blocked in 3\% non-fat dry milk for $1 \mathrm{~h}$ at room temperature, and probed with appropriate antibodies. Membranes were then probed with HRP-tagged anti-mouse or anti-rabbit IgG antibodies diluted 1:5,000-1:15,000 in 3\% non-fat dry milk for $1 \mathrm{~h}$ at room temperature. Chemiluminescence was detected using enhanced chemiluminescence (ECL).

Statistical analysis. Results are expressed as arithmetic mean \pm SEM (standard error of the mean). To compare the statistical significance between the groups, two-sided unpaired Student's t-test was used. All experiments were repeated three times and representative data are shown. Statistical analyses were performed using SPSS software (version 19.0; SPSS, Inc., Chicago, IL, USA). Mean differences with P-values $<0.05$ were considered to indicate a statistically significant result.

\section{Results}

Preparation and characterization of the MB-Lipo (Dox)IL4RTP complex. The gas (SF6)-filled MBs were prepared
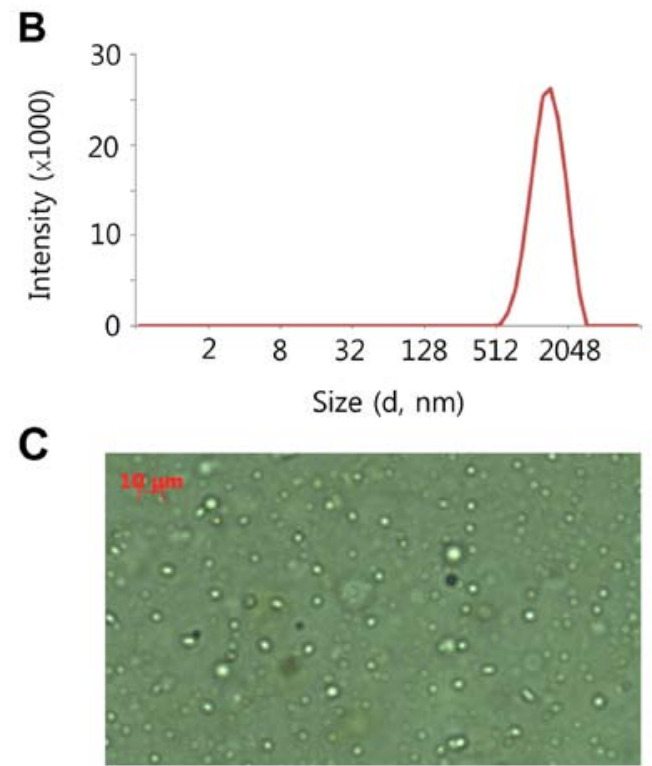

by emulsion and solvent-evaporation method. The prepared MBs were further conjugated with Dox-loaded nano-sized liposome and peptide ligands to IL4R for targeting brain tumor cells (Fig. 1A). The suspensions of the MBs in PBS appeared as milky white. Light microscopy revealed that the MBs were uniformly distributed with no visible aggregation. A single microbubble was round (Fig. 1C). As shown in Fig. 1B, the particle size ranged from 1,295-1,468 nm with a mean of 1,000 nm for MB-Lipo-IL4RTP and 1,000 nm for MB-Lipo. HPLC data demonstrated the incorporation of IL4R targeting peptide into MB-Lipo complex (yield, 75.6\%) (Fig. 1D).

Targeting ability of MB-Lipo-IL4RTP in U87MG cells. To select the most available brain tumor cell line with high expression of IL4R, we performed western blotting of IL4R in four brain tumor cell types (U87MG, LN229, HS683 and U138), one breast tumor cell line (MDA-MB-231) as positive control, and in one lung tumor cell line (H460). As shown in Fig. 2A, among brain tumor cell lines, U87MG cells had the highest expression of IL4R and H460 had 
A

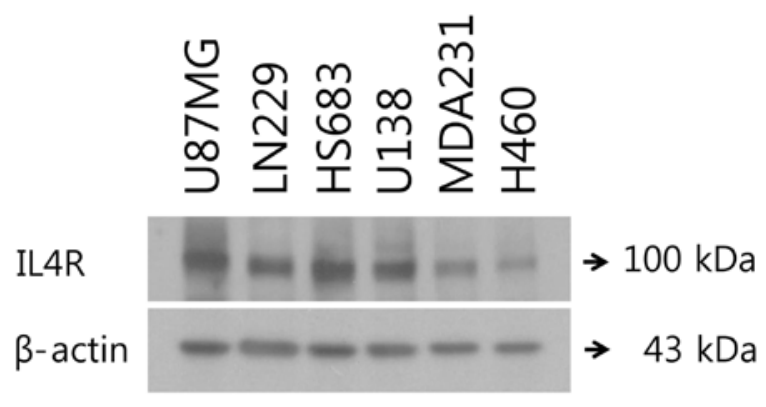

B

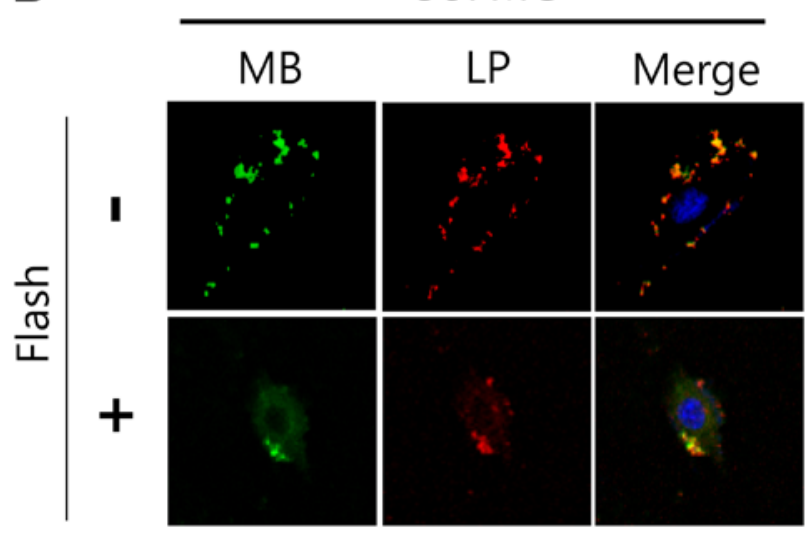

Figure 2. In vitro cancer cell targeting and intracellular delivery of MB-Lipo (Dox)-IL4RTP. (A) IL4R expression in the cancer cell lines. IL4R expression level in the brain tumor cells (U87MG, LN229, HS683 and U138), the breast cancer cells (MDA-MB-231) and the lung cancer cells (H460) was analyzed and compared by western blotting. IL4R expression of U87MG cells was pretty high. (B) Confocal laser scanning microscopy for binding and uptake of MB (FITC)-Lipo (Cy5)-IL4RTP into U87MG. Fluorescent MB (FITC)-Lipo (Cy5)-IL4RTP were used to label U87MG cells. MB (FITC)-Lipo (Cy5)-IL4RTP were mostly bound to cell membrane without flash and burst MBs and released Lipo particles with flash were taken up to the cytoplasm of cells as confirmed by green and red fluorescent signals inside the cells.

very low expression of IL4R. MBs targeted to IL4R were synthesized and binding specificity to IL4R was first tested in cell culture experiments. Fig. 2B illustrates binding of MB (FITC)-Lipo (Cy5) to IL4R-positive U87MG human brain tumor cells. IL $4 \mathrm{R}$ targeting peptide ligands on the surface of MB-Lipo complex target and bind to IL4R in U87MG brain tumor cells, which resulted in selective accumulation and long resident time in U87MG brain tumor cells. The MB-Lipo (Dox)-IL4RTP exhibited cellular uptake in U87MG brain tumor cells (one of brain tumor cell line expressing strongly IL4R) with frequency ultrasound energy suggesting that MB-Lipo (Dox)-IL4RTP provided effective targeting ability for brain tumor cells.

Cell growth inhibition effect of MB-Lipo (Dox)-IL4RTP against U87MG cells. For analysis of the effects of anticancer chemotherapy containing the ultrasound contrast agents to target cancer cells, and to evaluate the toxicity of the ultrasound contrast agents for ultrasound contrast media in the cells, WST-1 assay was performed (Fig. 3A and B). The cell viability measurements of U87MG (high IL4R expression)
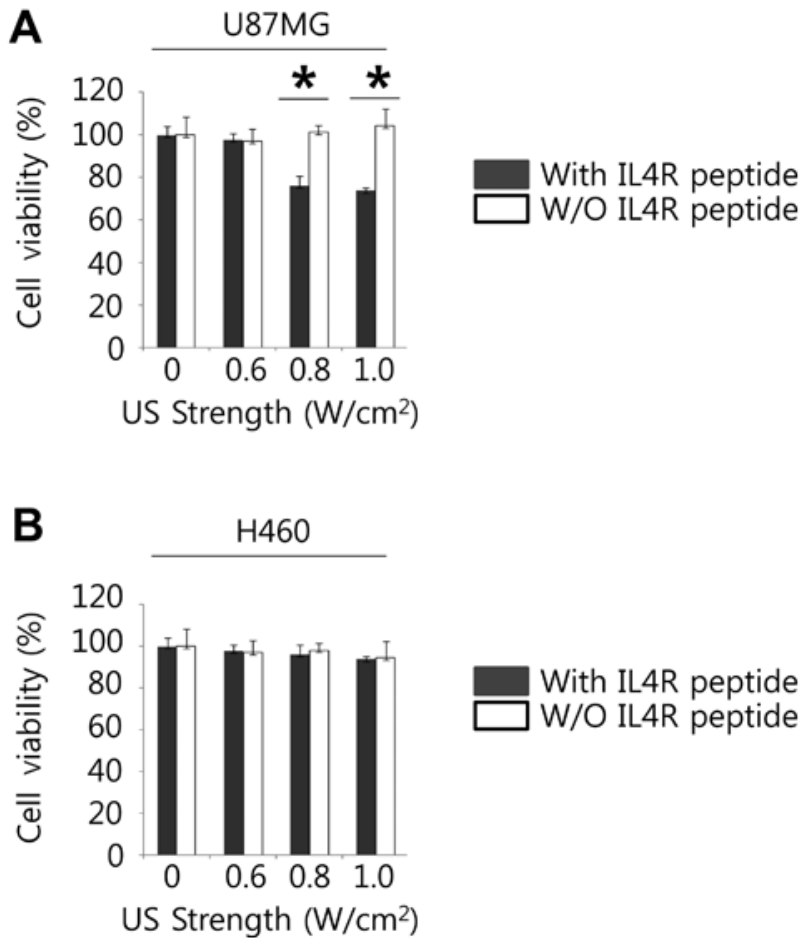

Figure 3. Inhibition of U87MG cell proliferation by MB-Lipo (Dox)-IL4RTP. (A) Incubation of U87MG (IL4R expression high) cells with MB-Lipo (Dox)IL4RTP caused the decreased cell proliferation under the 0.6 and $0.8 \mathrm{~W} / \mathrm{cm}^{2}$ of ultrasound strength. (B) Incubation of H460 (IL4R expression low) cells with MB-Lipo (Dox)-IL4RTP did not affect cell proliferation under the 0.6 and $0.8 \mathrm{~W} / \mathrm{cm}^{2}$ of ultrasound strength. The cell viability was determined by WST-1 assay and the relative cell survival rate percentage was calculated by dividing the optical density of each treatment condition by the optical density of the control (PBS) treatment. This result is representative of three biological replicates, and the error bars the mean standard deviation. ${ }^{*} \mathrm{P}<0.001$

with MB-Lipo (Dox)-IL4RTP (with IL4R targeting peptide ligands) were lower than MB-Lipo (Dox) (without IL4R targeting peptide ligands) $(\mathrm{P}<0.01)$ at the ultrasound strength of 0.8 and $1.0 \mathrm{w} / \mathrm{cm}^{2}$. However, the cell viability measurements of H460 (low IL4R expression) with MB-Lipo (Dox)-IL4RTP (with IL4R targeting peptide ligands) were almost the same as MB-Lipo (Dox) (without IL4R targeting peptide ligands) at the respective ultrasound strength of $0,0.6,0.8$ and $1.0 \mathrm{w} / \mathrm{cm}^{2}$. These results suggest that MB-Lipo (Dox)-IL4RTP may play an essential role in inhibiting cancer cell growth in an IL4R dependent manner.

DNA damaging response-related cell cycle arrest induced by MB-Lipo (Dox)-IL4RTP in U87MG cells. To examine the role of IL4R in MB-Lipo (Dox)-IL4RTP-induced DNA damaging response-related cell cycle arrest in U87MG cells, we incubated U87MG cells with MB-Lipo (Dox) with IL4R targeting peptide or MB-Lipo (Dox) without IL4R targeting peptide and analyzed the protein status of PARP1, Bax, phospho-p53 (Ser15), p53, p21 and $\gamma \mathrm{H} 2 \mathrm{AX}$ using western blot assays. MB-Lipo (Dox)-IL4RTP did not cause apoptosis in U87MG cells since PARP1 was not cleaved and Bax expression level was not changed. As shown in Fig. 4, MB-Lipo (Dox)-IL4RTP significantly augmented phospho-p53 (Ser15) and $\gamma \mathrm{H} 2 \mathrm{AX}$, two of the representative DNA damaging-related markers 


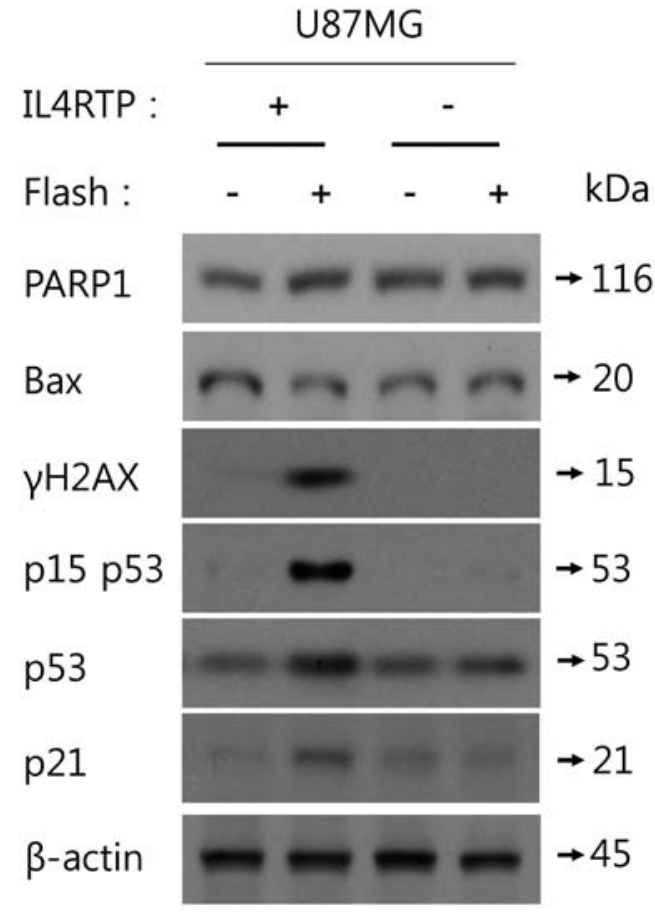

Figure 4.DNA damaging effect by doxorubicin from MB-Lipo (Dox)-IL4RTP in U87MG cells. Incubation of U87MG cells with MB-Lipo (Dox)-IL4RTP followed by flash induced upregulation of $\mathrm{p}(\mathrm{Ser} 15)-\mathrm{p} 53$ and $\mathrm{rH} 2 \mathrm{AX}$ that are representative DNA damaging markers, depending on IL4R targeting peptide. The levels of the indicated proteins were analyzed by western blotting. $\beta$-actin was used for a gel-loading control.

after ultrasound exposure in U87MG cells but MB-Lipo (Dox) could not affect them. In addition, MB-Lipo (Dox)-IL4RTP upregulated p53 and p21 expression that may activate cell cycle arrest. In contrast, MB-Lipo (Dox) did not change either p53 or p21 expression. Collectively, these results suggest that MB-Lipo (Dox)-IL4RTP may play an essential role in promoting IL4R-dependent cell cycle arrest after treatment and ultrasound exposure in U87MG cells.

\section{Discussion}

Ultrasound molecular imaging has great potential to impact early disease diagnosis, evaluation of disease progression and the development of target-specific therapy. In the present study, IL4R targeting peptide was conjugated onto the surface of microbubbles (MBs) to evaluate molecular imaging of brain tumors using U87MG cell line model. There have been studies on the high expression of IL4R in the various human solid tumors including brain tumors which promotes tumor development via upregulation of anti-apoptotic proteins and prevention of chemotherapy-induced cancer cell death. Thus, it would be a good therapeutic treatment to target IL4R on the brain tumor by developing a novel drug delivery system with IL4R targeted ligands. In addition, targeted therapy against cancer provides various biological advantages such as increase in the pharmacokinetic profile of drugs and minimization of the unexpected side-effect caused by the chemotherapy with high specificity.

In the present study, we designed an IL4R targeting peptide that was described as an atherosclerotic plaque and breast tumor tissue homing peptide screened by phage display screening method. According to the study using this peptide ligand (22), polymer conjugated with IL4R targeting peptide could play an important role in increasing in vitro tumor-specific targeting and uptake efficiency in both H226 and MDA-MB-231 cancer cell lines, and has excellent homing and longer retention in tumor tissues in MDA-MB-231 xenograft mouse model. These results suggested that IL4R targeting peptide ligands can be adapted to a novel tool for selective delivery of therapeutic drugs to tumors.

As one of the clinically most challenging fields, brain tumor exhibits various distinct characteristics. One of the obstacles to cure brain tumor is the blood-brain barrier ( $\mathrm{BBB})$ that is a blockade that blocks not only the external infectious organisms, but also the treatment for disease from entering deeply into the brain (23-28). Thus, various studies have been developing possible approaches to enhance the penetration activity of the helpful pharmaceutical options through BBB. For this purpose, a new technique using ultrasound and MBs has been actively applied to degenerative brain disease and brain cancers, which can lead the drug-loaded MBs by the focused ultrasound to enter properly into the brain (29). Recently, Wei et al reported that they had invented a novel liposomal formulation to overcome BBB conjugated with dual peptide ligands that are stable from protease in the blood using D-form amino acids. One of the peptide ligands was for nicotine acetylcholine receptors on the $\mathrm{BBB}$ and other ligands were for integrin highly expressed on both of $\mathrm{BBB}$ and tumor cells (30). According to the above studies, their liposome loaded with doxorubicin (Dox) could effectively penetrate $\mathrm{BBB}$ and target brain tumor in vitro and in vivo.

Although we proved that our novel microbubble complex containing Dox-loaded nano-sized liposome can target brain tumor cells and inhibit tumor cell growth with IL4R-dependent manner, the present study still has a few limitations. First, the targeting ability of MB (FITC)Lipo (Cy5)-IL4RTP to U87MG brain tumor cells was not performed by considering the real flow conditions in blood vessels since it may be important to reflect in vivo exposure of MB (FITC)-Lipo (Cy5)-IL4RTP to shear stress as well as the dose-dependence of MB (FITC)-Lipo (Cy5)IL4RTP. Secondly, in vivo ultrasound imaging and therapeutic effect of MB-Lipo-IL4RTP should be assessed using both xenograft and orthotopic mouse models with brain tumor cell lines that have different levels of IL4R expression. In conclusion, the present study contribute to the effort of development of methodology to measure the contrast enhancement effect of the new ultrasound contrast agent to breast tumor, and to target the breast cancer cell line in vitro and in vivo studies.

\section{Acknowledgements}

The present study was supported by grant no. 13-2014-006 from the SNUBH Research Fund. This study was supported by the Basic Science Research Program through the National Research Foundation of Korea (NRF) funded by the Ministry of Education, Science and Technology (NRF-2014R1A6A3A 04054307). 


\section{References}

1. Ostrom QT, Gittleman H, Liao P, Rouse C, Chen Y, Dowling J, Wolinsky Y, Kruchko C and Barnholtz-Sloan J: CBTRUS statistical report: Primary brain and central nervous system tumors diagnosed in the United States in 2007-2011. Neuro Oncol 16 (Suppl 4): iv1-iv63, 2014.

2. Louis DN, Ohgaki H, Wiestler OD, Cavenee WK, Burger PC, Jouvet A, Scheithauer BW and Kleihues P: The 2007 WHO classification of tumours of the central nervous system. Acta Neuropathol 114: 97-109, 2007.

3. Ohgaki $\mathrm{H}$ and Kleihues P: Population-based studies on incidence, survival rates, and genetic alterations in astrocytic and oligodendroglial gliomas. J Neuropathol Exp Neurol 64 479-489, 2005

4. Arora RS, Alston RD, Eden TO, Estlin EJ, Moran A and Birch JM: Age-incidence patterns of primary CNS tumors in children, adolescents, and adults in England. Neuro Oncol 11: 403-413, 2009.

5. Lee CH, Jung KW, Yoo H, Park S and Lee SH: Epidemiology of primary brain and central nervous system tumors in Korea. J Korean Neurosurg Soc 48: 145-152, 2010.

6. Dobes M, Khurana VG, Shadbolt B, Jain S, Smith SF, Smee R, Dexter $M$ and Cook R: Increasing incidence of glioblastoma multiforme and meningioma, and decreasing incidence of Schwannoma (2000-2008): Findings of a multicenter Australian study. Surg Neurol Int 2: 176, 2011.

7. Gigineishvili D, Shengelia N, Shalashvili G, Rohrmann S, Tsiskaridze A and Shakarishvili R: Primary brain tumour epidemiology in Georgia: First-year results of a population-based study. J Neurooncol 112: 241-246, 2013.

8. Dunn GP, Rinne ML, Wykosky J, Genovese G, Quayle SN, Dunn IF, Agarwalla PK, Chheda MG, Campos B, Wang A, et al: Emerging insights into the molecular and cellular basis of glioblastoma. Genes Dev 26: 756-784, 2012.

9. Stupp R, Mason WP, van den Bent MJ, Weller M, Fisher B, Taphoorn MJ, Belanger K, Brandes AA, Marosi C, Bogdahn U, et al; European Organisation for Research and Treatment of Cancer Brain Tumor and Radiotherapy Groups; National Cancer Institute of Canada Clinical Trials Group: Radiotherapy plus concomitant and adjuvant temozolomide for glioblastoma. $\mathrm{N}$ Engl J Med 352: 987-996, 2005.

10. Wen PY and Kesari S: Malignant gliomas in adults. N Engl J Med 359: 492-507, 2008.

11. Husain SR, Kreitman RJ, Pastan I and Puri RK: Interleukin-4 receptor-directed cytotoxin therapy of AIDS-associated Kaposi's sarcoma tumors in xenograft model. Nat Med 5: 817-822, 1999.

12. Joshi BH, Leland P, Asher A, Prayson RA, Varricchio F and Puri RK: In situ expression of interleukin-4 (IL-4) receptors in human brain tumors and cytotoxicity of a recombinant IL-4 cytotoxin in primary glioblastoma cell cultures. Cancer Res 61 8058-8061, 2001.

13. Joshi BH, Leland P, Lababidi S, Varrichio F and Puri RK: Interleukin-4 receptor alpha overexpression in human bladder cancer correlates with the pathological grade and stage of the disease. Cancer Med 3: 1615-1628, 2014.

14. Shimamura T, Royal RE, Kioi M, Nakajima A, Husain SR and Puri RK: Interleukin-4 cytotoxin therapy synergizes with gemcitabine in a mouse model of pancreatic ductal adenocarcinoma. Cancer Res 67: 9903-9912, 2007.
15. Husain SR, Gill P, Kreitman RJ, Pastan I and Puri RK: Interleukin-4 receptor expression on AIDS-associated Kaposi's sarcoma cells and their targeting by a chimeric protein comprised of circularly permuted interleukin-4 and Pseudomonas exotoxin. Mol Med 3: 327-338, 1997.

16. Kawakami K, Leland P and Puri RK: Structure, function, and targeting of interleukin 4 receptors on human head and neck cancer cells. Cancer Res 60: 2981-2987, 2000.

17. Murata T, Taguchi J and Puri RK: Interleukin-13 receptor alpha' but not alpha chain: A functional component of interleukin-4 receptors. Blood 91: 3884-3891, 1998.

18. Hosoyama T, Aslam MI, Abraham J, Prajapati SI, Nishijo K, Michalek JE, Zarzabal LA, Nelon LD, Guttridge DC, Rubin BP, et al: IL-4R drives dedifferentiation, mitogenesis, and metastasis in rhabdomyosarcoma. Clin Cancer Res 17: 2757-2766, 2011.

19. Kohno M, Horibe T, Haramoto M, Yano Y, Ohara K, Nakajima O, Matsuzaki K and Kawakami K: A novel hybrid peptide targeting EGFR-expressing cancers. Eur J Cancer 47: 773-783, 2011.

20. Garland L, Gitlitz B, Ebbinghaus S, Pan H, de Haan H, Puri RK, Von Hoff D and Figlin R: Phase I trial of intravenous IL-4 pseudomonas exotoxin protein (NBI-3001) in patients with advanced solid tumors that express the IL-4 receptor. J Immunother 28: 376-381, 2005.

21. Yoon YI, Kwon YS, Cho HS, Heo SH, Park KS, Park SG, Lee SH, Hwang SI, Kim YI, Jae HJ, et al: Ultrasound-mediated gene and drug delivery using a microbubble-liposome particle system. Theranostics 4: 1133-1144, 2014.

22. Sarangthem V, Cho EA, Bae SM, Singh TD, Kim SJ, Kim S, Jeon WB, Lee BH and Park RW: Construction and application of elastin like polypeptide containing IL-4 receptor targeting peptide. PLoS One 8: e81891, 2013.

23. Serwer LP and James CD: Challenges in drug delivery to tumors of the central nervous system: An overview of pharmacological and surgical considerations. Adv Drug Deliv Rev 64: 590-597, 2012.

24. Gao $\mathrm{H}$ and Jiang X: Progress on the diagnosis and evaluation of brain tumors. Cancer Imaging 13: 466-481, 2013.

25. Gao H, Pang Z and Jiang X: Targeted delivery of nano-therapeutics for major disorders of the central nervous system. Pharm Res 30: 2485-2498, 2013.

26. Zhan C, Wei X, Qian J, Feng L, Zhu J and Lu W: Co-delivery of TRAIL gene enhances the anti-glioblastoma effect of paclitaxel in vitro and in vivo. J Control Release 160: 630-636, 2012.

27. Sarin H, Kanevsky AS, Wu H, Sousa AA, Wilson CM, Aronova MA, Griffiths GL, Leapman RD and Vo HQ: Physiologic upper limit of pore size in the blood-tumor barrier of malignant solid tumors. J Transl Med 7: 51, 2009.

28. Groothuis DR: The blood-brain and blood-tumor barriers: A review of strategies for increasing drug delivery. Neuro Oncol 2: 45-59, 2000.

29. Papademetriou IT and Porter T: Promising approaches to circumvent the blood-brain barrier: Progress, pitfalls and clinical prospects in brain cancer. Ther Deliv 6: 989-1016, 2015.

30. Wei X, Gao J, Zhan C, Xie C, Chai Z, Ran D, Ying M, Zheng P and Lu W: Liposome-based glioma targeted drug delivery enabled by stable peptide ligands. J Control Release 218: 13-21, 2015. 